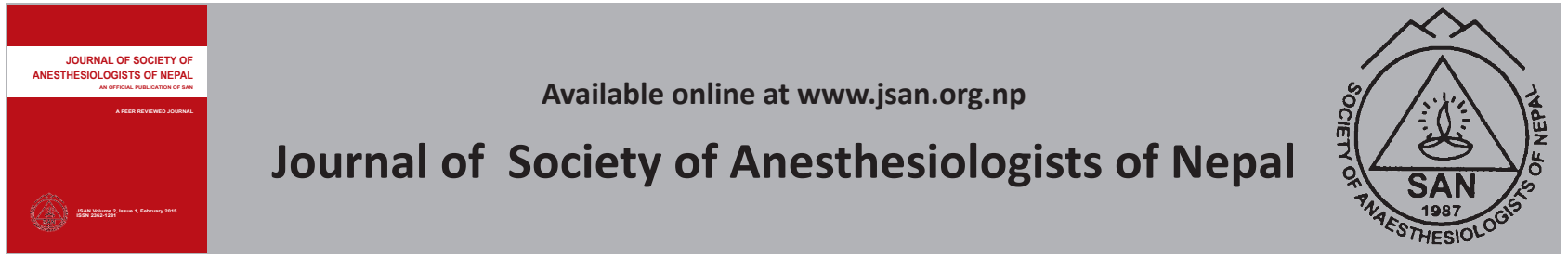

Original Article

\title{
In vitro study of Magnetic Resonance Imaging artifacts of anesthetic devices
}

Uday Bajracharya*, Prabhat Rawal**

*Shree Birendra Hospital, Chhauni and National Academy of Medical Sciences, Mahabaudha, Kathmandu, Nepal

**Nepal Armed Police Force Hospital, Balambu, Kathmandu, Nepal

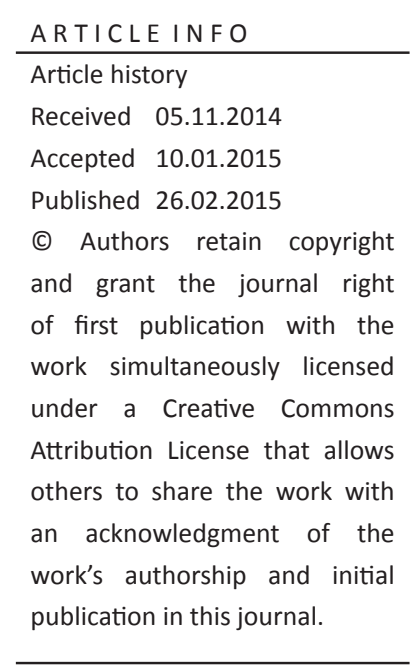

\begin{abstract}
Background: Deep sedation or general anesthesia is usually required for Magnetic Resonance Imaging when patients cannot remain motionless in the suite. Various anesthetic devices have been used to maintain the airway and ventilate the lungs during this period. Some of them produce artifacts that pose difficulties in the interpretation of images. The aim of this study was to identify the devices that produced artifacts during Magnetic Resonance Imaging.
\end{abstract}

Methods: Twelve anesthetic devices were considered: oro-pharyngeal airway, nasopharygeal airway, face mask with reservoir bag, nasal cannula, endotracheal tube, disposable Ambu Laryngeal Mask Airway, Laryngeal Mask Airway Unique, Disposable Laryngeal Tube Sonda, i-gel, Ambu bag, Bain Circuit, Jackson Rees Circuit. Magnetic Resonance Imaging was performed with each device placed on the top of a phantom simulator respectively to resemble the position in vivo.

Results: The artifacts with Disposable Laryngeal Tube Sonda, Laryngeal Mask Airway Unique and endotracheal tube were related to ferromagnetic material in the pilot valve and were similar. No artifacts were found with oro-pharyngeal airway, nasopharygeal airway, nasal cannula, endo-tracheal tube with pilot valve detached, face masks with reservoir bag (metal removed), Ambu bag (without Adjustable Pressure Limiting valve), i-gel, disposable Ambu Laryngeal Mask Airway, Bain Circuit and Jackson Rees Circuit.

Conclusion: Anesthetic devices that produce Magnetic Resonance Imaging artifacts are disposable Laryngeal Tube Sonda, Laryngeal Mask Airway Unique and Endotracheal Tube.

Key words: Anesthesia; artifacts; Magnetic Resonance Imaging

How to cite this article: Bajracharya U, Rawal P. In vitro study of Magnetic Resonance Imaging artifacts of anesthetic devices. JSAN 2015;2:13-16. 


\section{Introduction}

Deep sedation or generation anesthesia is usually required for Magnetic Resonance Imaging (MRI) when patients cannot remain motionless in the suite. ${ }^{1,2,3}$ During such conditions, the patient's airway is often maintained using airway adjuncts like Oropharyngeal Airway (OPA), Nasopharyngeal Airway (NPA), advanced airway devices like Endotracheal Tubes (ETT) and Laryngeal mask airway (LMA) and ventilation is managed using Bain circuit (BC) and Jackson Rees Circuit (JRC). Various artifacts are produced by these equipment during MRI scanning. ${ }^{4}$ OPA, NPA, face mask, $B C$, and JRC have no ferro-magnetic material in them but ETT, LMA and Disposable Laryngeal Tube Sonda (LTS-D) contain variable amount of ferromagnetic material that may reduce image quality. LMA ProSeal, LMA Flexible, LMA Fastrach and Flexible ETT have visible metal parts and certainly cause artifacts. ${ }^{5}$ There is little information available about the anesthetic equipment used during MRI scanning. Therefore, in vitro study of these devices would be useful to identify such artifacts as they pose a lot of difficulties in the interpretation of Magnetic Resonance (MR) images. The aim of this study was to identify the anesthetic devices that produced artifacts during Magnetic Resonance Imaging.

\section{Methods}

The study was approved by the Local Authority and Ethics Committee of the Institution. Twelve anesthetic devices were included. They were oropharyngeal airway (Guedel airway, Romsons ${ }^{\mathrm{TM}}$ ), nasopharygeal airway (Romsons ${ }^{\mathrm{TM}}$ ), oxygen face mask with reservoir bag (Hi mask, Romsons ${ }^{\mathrm{TM}}$ ), nasal prongs (Oxyset, Romsons ${ }^{\mathrm{TM}}$ ), endotracheal tube $\left(\right.$ Tyco $^{\mathrm{TM}}$ ), Laryngeal Mask Airways (Disposable Ambu LMA, LMA Unique ${ }^{T M}$ ), i-gel, Disposable Laryngeal tube Sonda $^{\mathrm{TM}}$ (BVM) (LTS-D), Ambu Bag without Adjustible Pressure Limiting (APL) valve (Laederal ${ }^{\mathrm{TM}}$ ), Bain circuit (Romsons $^{\mathrm{TM}}$ ) and Jackson Rees Circuit (Romsons ${ }^{\mathrm{TM}}$ ). They were evaluated during an MRI procedure in a 0.35-Tesla MRI scanner (Airis Elite Hitachi ${ }^{\mathrm{TM}}$ ).

Radiologists often use a Phantom Simulator (PS) to check the MRI machine for compatibility with implants and devices. A standard cylindrical water phantom made of polymethyl methacrylate plastic with dimensions of $12 \mathrm{~cm}$ x $24 \mathrm{~cm}$ (diameter $x$ height), filled with a nickel solution was placed in the center of the magnetic field where the head of a hypothetical patient would be positioned (Figure $0)$.The imaging planes were oriented in a standard way to encompass the short and long axis of the phantom using T-2 gradient-echo (GE) images: repetition time 1000 $\mathrm{ms}$; echo time $50 \mathrm{~ms}$; flip angle $20^{\circ}$, field of view $220^{\circ}$; Matrix 256 × 256. GE images were used because artifacts due to ferromagnetic objects are more prominent in GE sequences than spin-echo (SE) ones.
The anesthetic devices were placed on the top of the PS one at a time (Figures 1,2,3,4,5,6a,6b,7,8,9,10,11) such that the pilot balloon (if present) was positioned at a distance that simulated its actual position in vivo. The scans were repeated with each device to identify the presence of artifacts. The MRI scans were repeated with ETT, LMA Unique and LTS-D respectively, after removal of the pilot valve containing the metal spring. The artifacts of the MR images were subjectively evaluated by expert radiologists of different institutes.

The photographs of anesthetic devices on the Phantom Simulator (PS) are shown in the following figures (Fig):

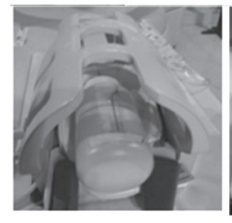

Fig.0

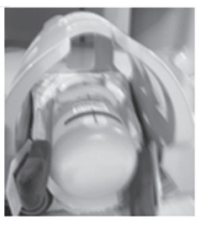

Fig.1

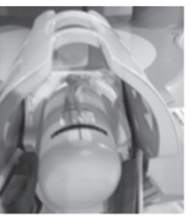

Fig.2

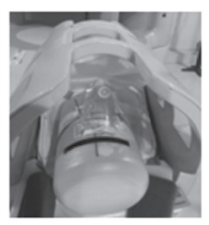

Fig.3
Fig 0. PS without anesthetic equipment

Fig 1. PS with Oropharyngeal Airway

Fig 2. PS with Nasopharyngeal airway

Fig 3. PS with Face mask with reservoir bag

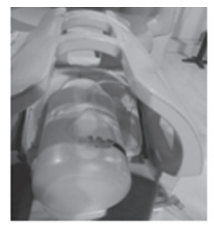

Fig.4

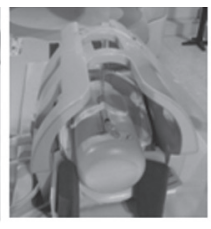

Fig.5

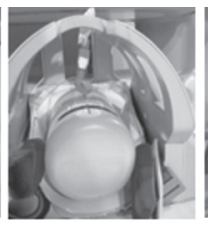

Fig.6a

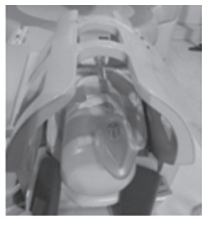

Fig.6b
Fig 4. PS with Nasal cannula

Fig 5. PS with Endotracheal Tube

Fig 6a. PS with Ambu LMA

Fig 6b. PS with LMA unique

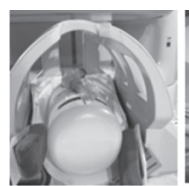

Fig.7

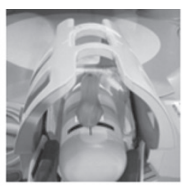

Fig.8

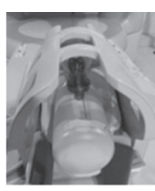

Fig.9

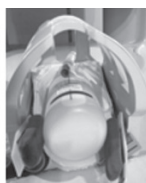

Fig.10

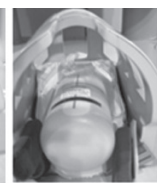

Fig.11
Fig 7. PS with Laryngeal Tube Sonda -D

Fig 8. PS with i-gel

Fig 9. PS with Ambu bag

Fig 10. PS with Bain circuit

Fig 11. PS with Jackson Rees circuit 


\section{Results}

The images of the different anesthesia equipment with T-2 GE sequences were obtained as shown in the figures below with the abbreviations of the names on them.

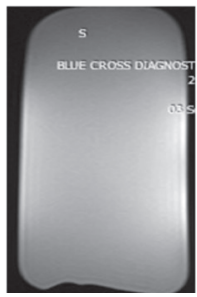

Fig.12

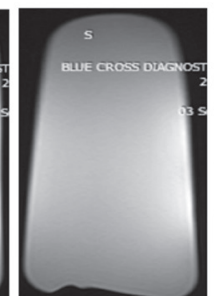

Fig.13

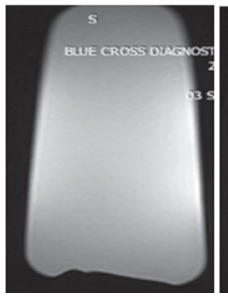

Fig.14

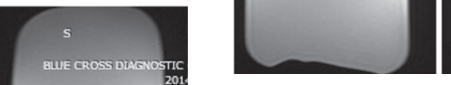

Fig. 24

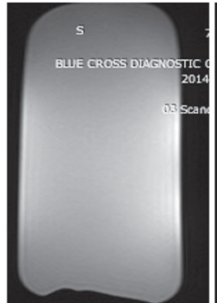

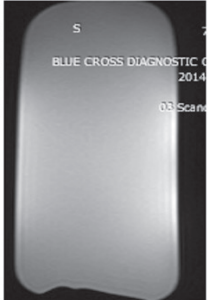

Fig. 25

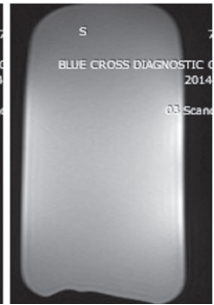

Fig.26

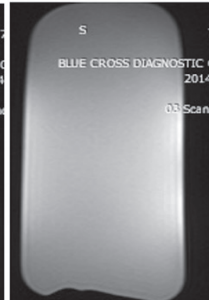

Fig. 27
24. i-gel

Fig 25. Ambu bag (without APL valve)

Fig 26. Bain Circuit

\section{Fig 27. Jackson Rees circuit JRC}

The images were studied for artifacts and they were observed with LTS-D, LMA Unique and ETT (with pilot valve). No artifacts were found with OPA, NPA, nasal cannula, face masks with reservoir bag (metal removed), Ambu bag (without Adjustable Pressure Limiting valve), i-gel, disposable Ambu LMA, Bain circuit and Jackson Rees circuit. No artifacts were noticed when the pilot valve was detached from ETT, LTS-D and LMA.

\section{Discussion}

Artifacts pose a lot of difficulties in image interpretation to the radiologists. Disposable LMA and other devices were used in this study. Reusable LMA and other equipment made of silicon are unsuitable for use in MRI because silicon being similar to human tissue can cause distortion of MR images and can also get heated up. ${ }^{6}$ However, Anez et $\mathrm{al}^{7}$ reported that the LMA ProSeal distorted MRI images (1-Tesla scan) but the classic LMA yielded acceptable images in a 4-year-old patient scheduled for a brain MRI. Steben and Burden ${ }^{8}$ found that the force exerted by the MRI magnet on an LMA-Flexible device was modest and that the cuff remained in place during the procedure (although the LMA-Flexible caused an artifact by producing a black hole around the tube). Its metallic spiral is an essential design that makes it flexible and is made up of a small mass of lightweight metal. This possibly explains the modest amount of magnetic force exerted on it such that it remains undisplaced from the magnetic field.

Our data for the twelve devices that yielded the artifacts is consistent with previous reports. The Ambu LMA disposable appears suitable for use during MRI. ${ }^{9}$ There are also data available on the use of the i-gel during MRI. ${ }^{10}$ The disposable circuits, face mask and airways also appear suitable for use during MRI. The magnetic susceptibility artifact is certainly more prominent with LMA ProSeal, Flexible LMA and LMA Fastrach. ${ }^{11,12}$ These devices were not evaluated for artifacts because they contain visible metal in them. 
The artifacts of the ETT, LMA Unique and LTS-D were similar and were by virtue of ferromagnetic material in the pilot balloon valve. Artifact may be seen in the case of Ambu bag due to the ferromagnetic material present in the spring of the Adjustable Pressure Limiting ( $A P L)$ valve but there were no artifacts when used without APL valve in the Ambu bag. MRI of ETT, LMA Unique and LTS-D were repeated after cutting away the pilot valve and the artifacts disappeared. There were no artifacts seen with OPA, NPA, face mask with the reservoir bag disposable Ambu LMA, i-gel and breathing circuits (BC, JRC).

Therefore, the artifacts were caused by the spring contained in the pilot balloon of the ETT, LMA Unique, and LTS-D, as this was the only metal part of those devices. ${ }^{13,14}$ This is supported by the fact that when the pilot balloon in those devices were removed, the artifacts disappeared. The OPA, NPA, face mask (externally seen metal removed), nasal cannula, Ambu LMA, i-gel, Bain circuit and Jackson Rees circuit do not contain any metal parts, so these airway devices may be more appropriate for use during MRI.

\section{Conclusion}

Anesthetic devices containing ferromagnetic material produce artifacts during Magnetic Resonance Imaging.

\section{Conflicts of interest: None}

Acknowledgements: We acknowledge the Department of Radiology, Shree Birendra Hospital, Blue Cross Diagnostic Centre, MRI Centre and Kathmandu Imaging, Mr DS Pujari, MRI center Naxal, Mr A Khadka, BCDC Bagbagar, Bishnu Tha Ma SBH Chhauni and H Yadav, KI, Maitighar, the technical staffs of Radiological department. Special thanks: Prof. RK Ghimire, HOD, Dept of Radiology, IOM; Dr D Shrestha, KMC; Dr KB Rawal and Prof Dr SB Basnet, SBH, Chhauni.

\section{References}

1. Gooden CK, Dilos B. Anesthesia for magnetic resonance imaging. Int Anesthesiol Clin 2003; 41:29-37.

2. Boutros A, Pavlicek W. Anesthesia for magnetic resonance imaging. Anesth Analg 1987; 66:367.

3. Osborn IP. Magnetic resonance imaging anesthesia: new challenges and techniques. Curr Opin Anaesthesiol 2002;15:443-8.

4. Crofts $S$, Campbell A. A source of artefact during general anaesthesia for magnetic resonance imaging. Anaesthesia 1993;48:643.

5. Fairfield JE. Laryngeal Mask and magnetic resonance- a caution. Anesthesia 1990;45:995.

6. Langton JA, Wilson I, Fell D. Use of the laryngeal mask airway during magnetic resonance imaging. Anesthesia 1992;47:532.

7. Anez C, Fuentes A, Jubera P, Sala JM, Tull M. The proseal laryngeal mask airway interferes with magnetic resonance imaging. Can J Anaesth 2005;52:116-7.
8. Stevens JE. Burden G. Reinforced laryngeal mask airway and magnetic resonance imaging. Anesthesia 1994;49:79-80.

9. Monclus E, Garces A, De Jose Maria B, Artes D, Mabrock M. Study of the adjustment of the Ambu laryngeal mask under magnetic resonance imaging. Pediatr Anesth 2007;17:1182-6.

10. Taxak S, Bhardwaj M, Gopinath A. The i-gel - A promising airway device for magnetic resonance imaging suite. J Anaesthesiol Clin Pharmacol 2012;28:263-4.

11. Schieble T, Patel A, Davidson M. Laryngeal mask airway (LMA) artefact resulting in MRI misdiagnosis. Pediatr Radiol 2008;38:328

12. Brimacombe JR. Intubating LMA for airway intubation. In: Brimacombe JR, editor. Laryngeal Mask Anesthesia: Principles and practice. 2nd ed. Philadelphia: Saunders; 2005. pp. 469-505.

13. Nixon C, Hirsch NP, Ormerod IEC, Johnson G. Nuclear Magnetic resonance; its implications for the anesthetist. Anaesthesia 1986;41:131-7.

14. Grady RE Jr, Perkins WJ. An unexpected cause of magnetic resonance image distortion: the endotracheal tube pilot balloon. Anesthesiology 1997;86:993-4. 